\title{
Picroside II inhibits hypoxia/reoxygenation-induced cardiomyocyte apoptosis by ameliorating mitochondrial function through a mechanism involving a decrease in reactive oxygen species production
}

\author{
JIAN-ZHE LI $^{1 *}$, SHU-YI YU $^{2 *}$, DAN MO $^{3}$, XIU-NENG TANG ${ }^{1}$ and QING-RUI SHAO ${ }^{1}$ \\ ${ }^{1}$ Department of Pharmacy, Ruikang Hospital, Guangxi University of Chinese Medicine, Nanning, Guangxi 530011; \\ ${ }^{2}$ Modern Analysis and Testing Center, Central South University, Changsha, Hunan 410078; ${ }^{3}$ Department of Surgery, \\ Maternal and Child Health Hospital of the Guangxi Zhuang Autonomous Region, Nanning, Guangxi 530003, P.R. China
}

Received July 26, 2014; Accepted November 21, 2014

DOI: $10.3892 /$ ijmm.2014.2009

\begin{abstract}
Reactive oxygen species (ROS)-induced mitochondrial dysfunction plays an important role in cardiomyocyte apoptosis during myocardial ischemia/reperfusion (I/R) injury. Picroside II, isolated from Picrorhiza scrophulariiflora Pennell (Scrophulariaceae), has been reported to protect cardiomyocytes from hypoxia/reoxygenation (H/R)-induced apoptosis, but the exact mechanism is not fully clear. The aim of the present study was to explore the protective effects of picroside II on $\mathrm{H} / \mathrm{R}$-induced cardiomyocyte apoptosis and the underlying mechanism. In the $\mathrm{H} 9 \mathrm{c} 2$ rat cardiomyocyte cell line, picroside II $(100 \mu \mathrm{g} / \mathrm{ml})$ was added for $48 \mathrm{~h}$ prior to $\mathrm{H} / \mathrm{R}$. The results showed that picroside II markedly inhibited $\mathrm{H} / \mathrm{R}$-induced cardiomyocyte apoptosis. In addition, picroside II was also able to decrease the opening degree of mitochondrial permeability transition pore (mPTP), increase the mitochondrial membrane potential, inhibit cytochrome $c$ release from mitochondria to cytosol and downregulate caspase-3 expression and activity concomitantly with the decreased ROS production. These results suggested that picroside II inhibited H/R-induced cardiomyocyte apoptosis by ameliorating mitochondrial function through a mechanism involving a decrease in ROS production.
\end{abstract}

Correspondence to: Dr Shu-Yi Yu, Modern Analysis and Testing Center, Central South University, 110 Xiang-Ya Road, Changsha, Hunan 410078, P.R. China

E-mail: shuyi_yu0731@126.com

${ }^{*}$ Contributed equally

Key words: picroside II, hypoxia/reoxygenation, cardiomyocytes, apoptosis, mitochondria, reactive oxygen species

\section{Introduction}

Myocardial ischemia/reperfusion (I/R) injury refers to cardiac dysfunction induced by the restoration of blood flow following a period of ischemia or lack of oxygen (1). Myocardial I/R injury is a common clinical problem and frequently found in the process of coronary thrombolysis, coronary artery bypass graft and heart transplantation (2-4). Cardiomyocyte apoptosis is well-established as one of the major mechanisms for I/R-induced injury $(5,6)$. Currently, the majority of studies supported that I/R-induced cardiomyocyte apoptosis was closely associated with excessive production of reactive oxygen species (ROS) $(7,8)$.

ROS are chemically-reactive molecules containing the element oxygen, including superoxide anion $\left(\mathrm{O}_{2}{ }^{-}\right)$, hydroxyl radical $(\bullet \mathrm{OH})$ and hydrogen peroxide $\left(\mathrm{H}_{2} \mathrm{O}_{2}\right)(9)$. Under physiological conditions, there is a low concentration of ROS in the heart. They are mainly produced as a by-product of mitochondrial oxidative phosphorylation. However, multiple pathological conditions, including $\mathrm{I} / \mathrm{R}$, can result in the accumulation of ROS (10,11). Mitochondrion has been reported as the main target of ROS damage (12). By contrast, increased ROS can directly lead to the oxidative damage of mitochondrial DNA, membrane phospholipids and respiratory chain proteins $(13,14)$. Increased ROS are able to enhance the opening degree of mitochondrial permeability transition pore (mPTP) (15). The opening of mPTP leads to the increase in mitochondrial permeability, mitochondrial swelling, mitochondrial membrane potential collapse and cytochrome $c$ release from mitochondria to cytosol, which ultimately results in cardiomyocyte apoptosis (16). The above studies suggested that ROS-induced mitochondrial dysfunction plays an important role in cardiomyocyte apoptosis and a decrease in ROS production may be a promising strategy for the inhibition of I/R-induced cardiomyocyte apoptosis.

Picroside II ( $\beta$-D-glucopyranoside, 1a,1b,2,5a,6,6ahexahydro-6-[(4-hydroxy-3-methoxybenzoyl)oxyl]-1a-(hydroxy methyl)oxireno[4,5]cyclopenta[1,2-c]pyran-2-yl) is a primary active ingredient extracted from Picrorhiza scrophulariiflora 
Pennell (Scrophulariaceae), which is a traditional Chinese herbal medicine and has been extensively used in China for $>1000$ years (17). In recent years, it has been reported that picroside II has an antioxidant property. The study by Li et al (18) demonstrated that pretreatment of PC12 cells with picroside II significantly prevented glutamate-induced cell apoptosis by inhibition of ROS production. In addition, treatment with picroside II evidently ameliorated liver damage induced by carbon tetrachloride, D-galactosamine and acetaminophen. The hepatoprotective effect of picroside II was associated with scavenging free radicals and protecting normal constructions of mitochondria membrane (19). Recently, it has been reported that picroside II has the protective effect on H/R-induced cardiomyocyte apoptosis (20), but the exact mechanism is not fully understood.

The present study aimed to investigated the important role of ROS-induced mitochondrial dysfunction in cardiomyocyte apoptosis during $\mathrm{I} / \mathrm{R}$ and the antioxidant property of picroside II. Using the hypoxia/reoxygenation (H/R) model in the H9c2 cardiomyocyte cell line to simulate I/R injury, the study explored whether picroside II has a protective effect on H/R-induced cardiomyocyte apoptosis, and whether the protective effect is associated with ameliorating mitochondrial function by inhibition of ROS production.

\section{Materials and methods}

Materials. H9c2 embryonic rat heart-derived cells were obtained from Academia Sinica (Shanghai, China). Picroside II (purity $>99 \%$ ) was purchased from the Chinese National Institute for the Control of Pharmaceutical and Biological Products (Beijing, China), and dissolved in sterile distilled water. Dulbecco's modified Eagle medium (DMEM) and fetal bovine serum (FBS) were provided from Gibco RBL (Grand Island, NY, USA). Trizol reagent was a product of Invitrogen Life Technologies (Carlsbad, CA, USA). The First Strand cDNA Synthesis kit was purchased from MBI Fermentas, Inc. (Vilnius, Lithuania). Cytochrome $c$ and $\beta$-actin antibodies were from Abcam (Cambridge, UK). The 3-(4,5-dimethylthiazol-2-yl)-2,5-diphenyl tetrazolium bromide (MTT), Hoechst 33342, caspase-3 activity assay kits and reactive oxygen species (ROS) detection kit were from Beyotime Biotechnology (Jiangsu, China). Lactate dehydrogenase $(\mathrm{LDH})$ and creatine kinase $(\mathrm{CK})$ assay kits were obtained from Nanjing Jiancheng Bioengineering Institute (Nanjing, China)

Cells culture and treatment. $\mathrm{H} 9 \mathrm{c} 2$ cells were cultured in DMEM supplemented with $15 \%$ (v/v) FBS, $100 \mathrm{U} / \mathrm{ml}$ penicillin and $100 \mu \mathrm{g} / \mathrm{ml}$ streptomycin at $37^{\circ} \mathrm{C}$ in a humidified atmosphere of $5 \% \mathrm{CO}_{2}$, with medium changed every 2-3 days. The cells were passaged regularly. When they reached $80 \%$ confluence, cells were made quiescent by serum starvation $(0.5 \%$ FBS) for $12 \mathrm{~h}$, and subsequently were treated with picroside II $(100 \mu \mathrm{g} / \mathrm{ml})$ for $48 \mathrm{~h}$ prior to H/R. For the H/R experiment, a hypoxic incubator was used to produce an in vitro hypoxia challenge. Cells in the hypoxic incubator were subjected to hypoxia $\left(<0.5 \% \mathrm{O}_{2}\right)$ using pre-conditioned hypoxic medium [ $8 \mathrm{~g} \mathrm{NaCl}, 0.4 \mathrm{~g} \mathrm{KCl}, 0.14 \mathrm{~g} \mathrm{CaCl}_{2}, 0.2 \mathrm{~g} \mathrm{MgSO}_{4} \cdot 7 \mathrm{H}_{2} \mathrm{O}, 1.2 \mathrm{~g}$ $\left.\mathrm{K}_{2} \mathrm{HPO}_{4} \cdot 12 \mathrm{H}_{2} \mathrm{O}, 0.08 \mathrm{~g} \mathrm{KH}_{2} \mathrm{PO}_{4}(\mathrm{pH} 7.4)\right]$ at $37^{\circ} \mathrm{C}$. Hypoxic medium was changed to fresh DMEM medium upon initiation of reoxygenation. The cells were subsequently cultured in the incubator under an atmosphere of $5 \%(\mathrm{v} / \mathrm{v}) \mathrm{CO}_{2}$ at $37^{\circ} \mathrm{C}$. The selected period of hypoxia and reoxygenation time was based on the preliminary time course studies. The most suitable period of $\mathrm{H} / \mathrm{R}$ was found to be $3 \mathrm{~h}$ hypoxia followed by $12 \mathrm{~h}$ reoxygenation. Control cells were cultured in normoxic conditions.

Evaluation of cell injury. Cardiomyocyte viability was measured by the MTT quantitative colorimetric assay. After picroside II treatment and $\mathrm{H} / \mathrm{R}$, the medium was removed and the cardiomyocytes were washed twice with phosphate-buffered saline (PBS), subsequently $10 \mu \mathrm{l}$ MTT solution was added to each well and incubated for an additional $4 \mathrm{~h}$ at $37^{\circ} \mathrm{C}$. Following this, $100 \mu \mathrm{l}$ dimethysulfoxide was added to dissolve the formazan product. The absorbance at $490 \mathrm{~nm}$ was read on a microplate reader and the data were expressed as a percentage of the control, which was considered $100 \%$ viable. $\mathrm{CK}$ and $\mathrm{LDH}$, the indicators of cardiomyocyte injury, were detected by the commercially available colorimetric assay kits according to the manufacturer's instructions.

Apoptosis analysis. Apoptotic cells were determined by fluorescent microscopy using the fluorescent dye Hoechst 33342. Briefly, H9c2 cells were seeded at a density of $1 \times 10^{5}$ cells/well in 12-well plates and cultured as described above. Following picroside II treatment and $\mathrm{H} / \mathrm{R}$, the medium was aspirated and the cells were washed twice with PBS. Subsequently, Hoeshst 33342 solution $(0.1 \mathrm{mg} / \mathrm{ml})$ was added into each well of the 12 -well plate for $20 \mathrm{~min}$ at $37^{\circ} \mathrm{C}$ in dark, followed by another three washes with PBS. Nuclear DNA staining was observed by a fluoresence microscope at $521 \mathrm{~nm}$ emission wavelength. The percent of apoptosis was expressed as a ratio of apoptotic to total cells. Caspase-3 is a key enzyme in the apoptosis cell-signaling cascade. The activity of caspase-3 was measured by the commercially available colorimetric assay kit according to the manufacturer's instructions. The data were expressed as a percentage of the control.

Quantitative polymerase chain reaction ( $q P C R)$. Following picroside II treatment and $\mathrm{H} / \mathrm{R}$, total RNA was isolated from the $\mathrm{H} 9 \mathrm{c} 2$ cells by the Trizol reagent and quantified by measuring the absorbance at $260 \mathrm{~nm}$. A $1 \mu \mathrm{g}$ RNA aliquot from each sample was reverse-transcripted to cDNA using the M-MLV Reverse transcriptase kit. The cDNA was used for qPCR. Quantitative analysis of mRNA expression was performed by the ABI 7300 real-time PCR system with the Power SYBR Green PCR Master Mix kit. PCR primers were as follows: caspase-3 primers (sense, 5'-CAAGTCGATGGACTCTGGAA-3' and anti-sense, 5'-GTACCATTGCGAGCTGACAT-3'); GAPDH primers (sense, 5'-TGGCCTCCAAGGAGTAAGAAAC-3' and anti-sense, 5'-GGCCTCTCTCTTGCTCTCAGTATC-3'). The PCR amplification profiles consisted of denaturation at $95^{\circ} \mathrm{C}$ for $10 \mathrm{~min}$, followed by 40 cycles of denaturation at $95^{\circ} \mathrm{C}$ for $15 \mathrm{sec}$ and annealing at $60^{\circ} \mathrm{C}$ for $60 \mathrm{sec}$. All the amplification reactions for each sample were carried out 4 times, and the relative expression values were normalized to the expression value of $G A P D H$. 
Preparation of subcellular fractions. H9c2 cells were cultured as described above. Following picroside II treatment and H/R, the cells were collected and the preparation of subcellular fractions was isolated using the Cell Mitochondria Isolation kit. The method was carried out according to the manufacturer's instructions. Briefly, cells were washed in PBS and centrifuged at $600 \mathrm{x} \mathrm{g}$ for $10 \mathrm{~min}$ at $4^{\circ} \mathrm{C}$. The supernatant was removed and the cells were resuspended in the mitochondria isolation reagent. Following a $15 \mathrm{~min}$ incubation on ice, lysates were homogenized and centrifuged at $600 \mathrm{xg}$ for $10 \mathrm{~min}$ at $4^{\circ} \mathrm{C}$. The supernatant was subsequently centrifuged at $11,000 \mathrm{x}$ g for $10 \mathrm{~min}$ to isolate the mitochondria fraction in the pellet, while the supernatant was used to isolate the cytosol. The supernatant was subjected to centrifugation at 12,000 $\mathrm{x}$ g for $10 \mathrm{~min}$. The resulting supernatant was designated as the cytosolic fraction, which was used for the detection of cytochrome c.

Measurement of intracellular ROS. The levels of ROS were measured by oxidative conversion of cell permeable 2',7'-dichlorofluorescein diacetate to fluorescent dichlorofluorescein. $2^{\prime}, 7^{\prime}$-dichlorofluorescein was added at a final concentration of $10 \mu \mathrm{mol} / 1$ and incubated for $20 \mathrm{~min}$ at $37^{\circ} \mathrm{C}$. Fluorescence intensity was detected using a fluorescence plate reader at an excitation wavelength at $488 \mathrm{~nm}$ and an emission wavelength at $525 \mathrm{~nm}$. The results were expressed as a percentage of the control.

Measurement of mPTP opening. The MPTP opening of cardiomyocytes was measured with calcein-acetoxymethylester (calcein-AM) in the presence of cobalt chloride using the mPTP fluorescence assay kit according to the manufacturer's instructions. In brief, cardiomyocytes were washed with Reagent $\mathrm{A}$, and subsequently the cells were incubated with Reagents $\mathrm{B}$ and $\mathrm{C}$ for $20 \mathrm{~min}$ at $37^{\circ} \mathrm{C}$, prior to washing twice with Reagent A again. Fluorescence intensity was determined by a SprctraMax M5 microplate reader at an excitation wavelength at $488 \mathrm{~nm}$ and an emission wavelength at $505 \mathrm{~nm}$. Fluorescence intensity was normalized to total protein concentration in the corresponding cell and the data were expressed as normalized relative fluorescence units (U/mg protein).

Determination of mitochondrial membrane potential. Mitochondrial membrane potential was determined by the tetramethylrhodamine ethyl ester (TMRE) fluorescent dye. TMRE is positively charged and highly permeable across the mitochondrial membrane. Mitochondrial depolarization is able to result in the spread of TMRE from mitochondria to cytoplasm, and enhance the whole-cell fluorescence intensity. TMRE was added at a final concentration of $1 \times 10^{-7} \mathrm{~mol} / 1$ and incubated in the dark for $20 \mathrm{~min}$ at room temperature. Fluorescence intensity was determined (excitation wavelength at $514 \mathrm{~nm} / \mathrm{emission}$ wavelength at $590 \mathrm{~nm}$ ). The data were expressed as a percentage of the control.

Western blotting analysis. Protein concentration was determined by a Bradford protein assay. Equal amounts of protein were separated by $10 \%$ sodium dodecyl sulfate-polyacrylamide gel electrophoresis and transferred to a nitrocellulose membrane. After being blocked with $5 \%$ milk powder in TBST for $1 \mathrm{~h}$ at room temperature, the membranes were incubated
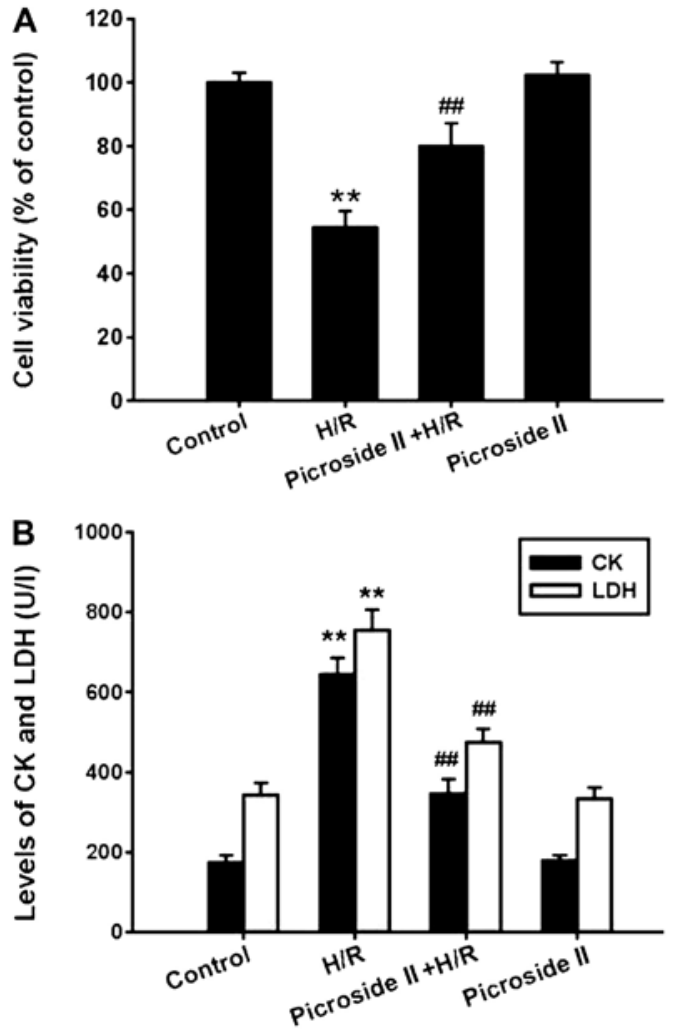

Figure 1. Effect of picroside II on H/R-induced cell injury in H9c2 cells. (A) Cell viability and (B) levels of CK and LDH. Data are expressed as mean \pm standard error of the mean and represent four independent experiments. ${ }^{* *} \mathrm{P}<0.01$ vs. control; ${ }^{\# \#} \mathrm{P}<0.01$ vs. H/R. H/R, hypoxia/reoxygenation; $\mathrm{CK}$, creatine kinase; LDH, lactate dehydrogenase.

with the primary antibody for cytochrome $c$ (diluted $1 \mu \mathrm{g} / \mathrm{ml}$, Cat. no. ab90529) and $\beta$-actin (diluted 1:1,000, Cat. no. ab1801) (both rabbit polyclonal antibodies; Abcam) at $4^{\circ} \mathrm{C}$ overnight, and followed by incubation with the corresponding horseradish peroxidase-conjugated secondary antibody (goat anti-rabbit; (diluted 1:5,000, Cat. no. ab175773; Abcam) at room temperature for $1 \mathrm{~h}$. Detection was performed using an ECL kit according to the manufacturer's instructions. The results were normalized to $\beta$-actin expression.

Statistical analysis. Data are expressed as the means \pm standard error of the mean. All the values were analyzed using analysis of variance and the Newman-Keuls Student's $t$-test. $\mathrm{P}<0.05$ was considered to indicate a statistically significant difference.

\section{Results}

Effect of picroside II on H/R-induced cell injury. The MTT assay showed that the cell viability was significantly decreased after hypoxia for $3 \mathrm{~h}$ and reoxygenation for $12 \mathrm{~h}$. Pretreatment with picroside II for $48 \mathrm{~h}$ prior to $\mathrm{H} / \mathrm{R}$ was able to markedly inhibit the decrease in cell viability (Fig. 1A). In line with the MTT assay, the levels of CK and LDH, which are the indicators of cardiomyocyte injury, were significantly increased in the H/R group, which was also inhibited by pretreatment of picroside II (Fig. 1B). However, picroside II alone had no effect on cell viability and the production of CK and LDH. 


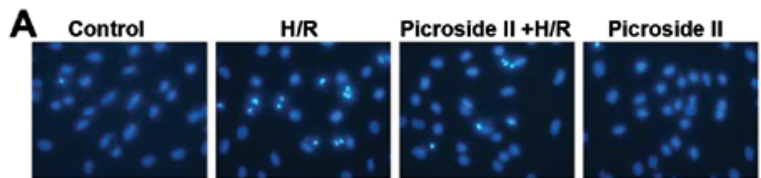

$\mathbf{B}$

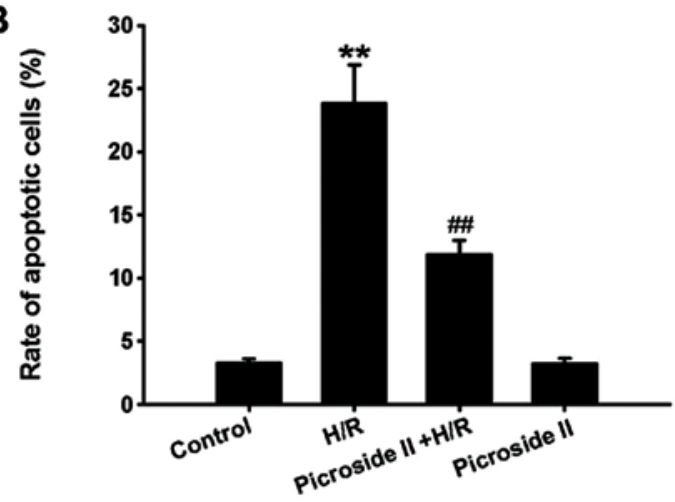

Figure 2. Effect of picroside II on H/R-induced cell apoptosis in H9c2 cells. (A) Representative image of Hoechst staining. (B) Rate of apoptotic cells. Data are expressed as mean \pm standard error of the mean and represent four independent experiments. ${ }^{* *} \mathrm{P}<0.01$ vs. control; ${ }^{\# \#} \mathrm{P}<0.01$ vs. H/R. H/R, hypoxia/reoxygenation.
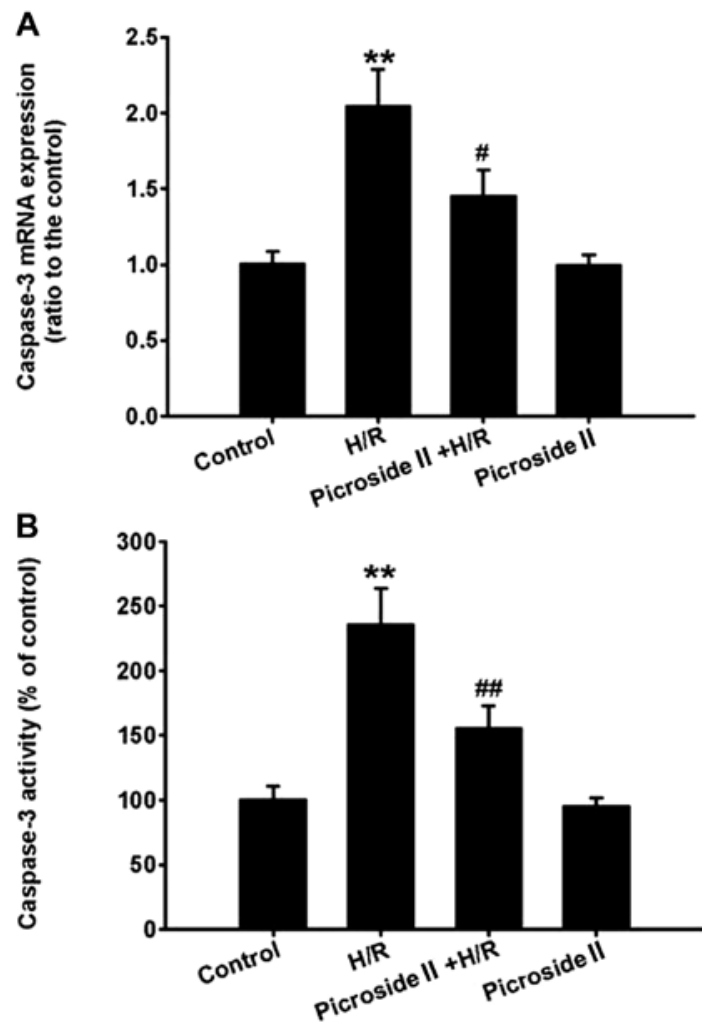

Figure 3. Effect of picroside II on H/R-induced caspase-3 mRNA expression and activity in H9c2 cells. (A) Caspase-3 mRNA expression and (B) caspase-3 activity. Data are expressed as mean \pm standard error of the mean and represent four independent experiments. ${ }^{* *} \mathrm{P}<0.01$ vs. control; ${ }^{~} \mathrm{P}<0.05$, ${ }^{\# \prime} \mathrm{P}<0.01$ vs. H/R. H/R, hypoxia/reoxygenation.

Effect of picroside II on H/R-induced cell apoptosis. The Hoechst 33342 staining assay showed that the percentage of apoptotic cells was significantly increased after hypoxia

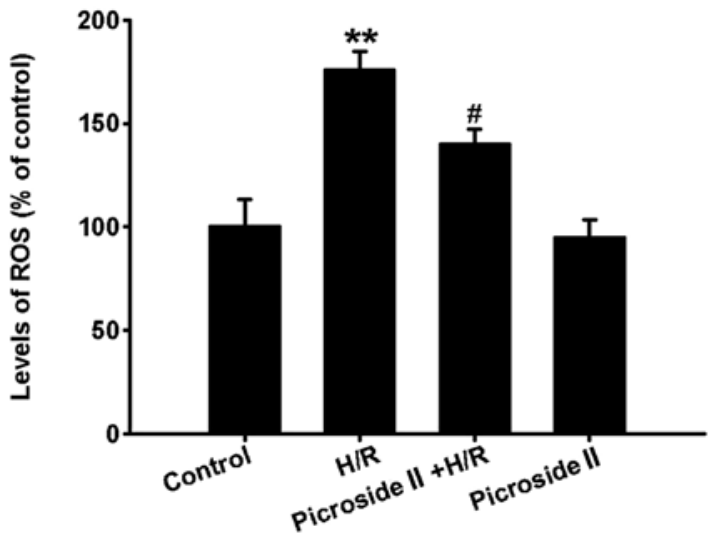

Figure 4. Effect of picroside II on H/R-induced intracellular ROS production in $\mathrm{H} 9 \mathrm{c} 2$ cells. Data are expressed as mean \pm standard error of the mean and represent four independent experiments. ${ }^{* *} \mathrm{P}<0.01$ vs. control; ${ }^{*} \mathrm{P}<0.05$ vs. H/R. H/R, hypoxia/reoxygenation; ROS, reactive oxygen species.

for $3 \mathrm{~h}$ and reoxygenation for $12 \mathrm{~h}$ compared to the control group. Consistent with the results of Hoechst 33342 staining, the mRNA expression and activity of caspase-3, the effector caspase of the mitochondrial apoptosis pathway, were also markedly increased in the H/R group. These effects induced by $H / R$ were significantly inhibited by pretreatment of picroside II (Figs. 2 and 3). However, picroside II alone had no effect on cardiomyocyte apoptosis and the mRNA expression and activity of caspase-3.

Effect of picroside II on H/R-induced intracellular ROS production. Compared to the control group, the levels of intracellular ROS were clearly increased after hypoxia for $3 \mathrm{~h}$ and reoxygenation for $12 \mathrm{~h}$, which was indicated by the increased fluorescence intensity. However, pretreatment of picroside II significantly inhibited the production of intracellular ROS induced by H/R (Fig. 4). Picroside II alone had no effect on the production of intracellular ROS.

Effect of picroside II on H/R-induced mPTP opening. The opening of MPTP was determined with calcein-AM in the presence of cobalt chloride. Calcein-AM fluorescent dye is able to move freely between mitochondrial and cytosol via the opening of mPTP. Cobalt chloride can quench calcein-AM fluorescence, but it cannot eliminate calcein-AM fluorescence in mitochondria when the mPTP closed. Due to this property, the fluorescence intensity of calcein-AM following treatment with cobalt chloride can reflect the extent of mPTP opening. The results showed that the fluorescence intensity was significantly decreased in the $H / R$ group, indicating that the extent of mPTP opening is enhanced following $H / R$. However, pretreatment of picroside II was able to markedly increase the fluorescence intensity compared to the H/R group (Fig. 5). Picroside II alone had no effect on the fluorescence intensity. These results indicated that picroside II decreased the degree of $\mathrm{MPTP}$ opening in response of H/R-induced injury.

Effect of picroside II on mitochondrial membrane potential after $H / R$. The results showed that mitochondrial membrane 


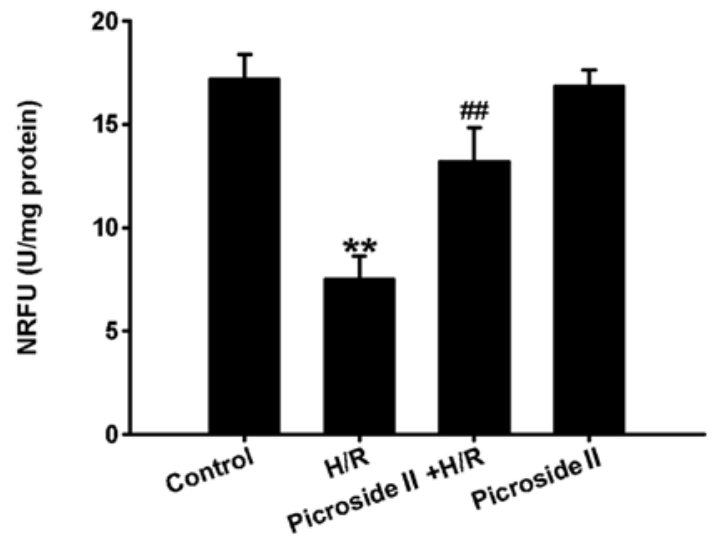

Figure 5. Effect of picroside II on H/R-induced opening of mPTP in H9c2 cells. Data are expressed as mean \pm standard error of the mean and represent four independent experiments. ${ }^{* *} \mathrm{P}<0.01$ vs. control; ${ }^{\# \#} \mathrm{P}<0.01$ vs. H/R. H/R, hypoxia/reoxygenation; $\mathrm{mPTP}$, mitochondrial permeability transition pore.

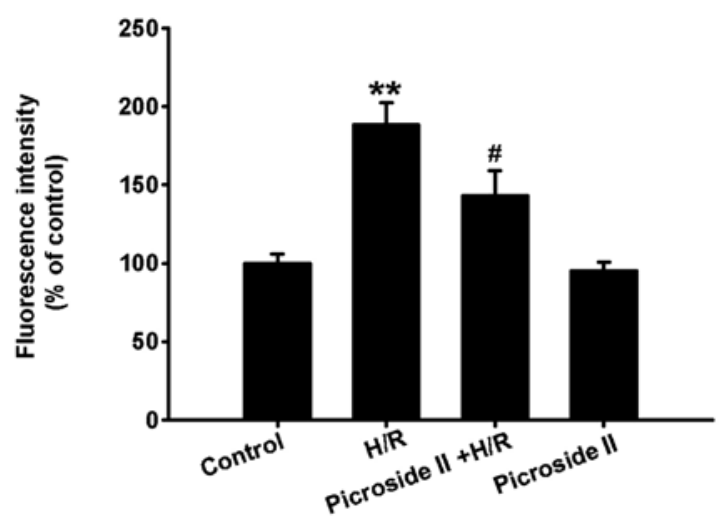

Figure 6. Effect of picroside II on H/R-induced mitochondrial membrane potential in $\mathrm{H} 9 \mathrm{c} 2$ cells. Data are expressed as mean \pm standard error of the mean and represent four independent experiments. ${ }^{* *} \mathrm{P}<0.01$ vs. control; ${ }^{\sharp} \mathrm{P}<0.05$ vs. H/R. H/R, hypoxia/reoxygenation.

potential was depolarized after hypoxia for $3 \mathrm{~h}$ and reoxygenation for $12 \mathrm{~h}$, which was indicated by the increased fluorescence intensity of TMRE. Pretreatment of picroside II was able to markedly decrease the fluorescence intensity of TMRE compared to the H/R group (Fig. 6). However, picroside II alone had no effect on the fluorescence intensity.

Effect of picroside II on H/R-induced mitochondrial cytochrome c release. In mitochondria, there was a high level of cytochrome $c$ expression in the control group. However, the protein expression of cytochrome $c$ in mitochondria was notably decreased following H/R. This H/R-induced effect was significantly inhibited by pretreatment of picroside II. By contrast, the cytochrome $c$ expression in the cytosol was lower in the control group compared to the H/R group. Pretreatment of picroside II was able to significantly reduce the increase in cytosolic cytochrome $c$ expression induced by H/R (Fig. 7). Picroside II alone had no effect on cytochrome $c$ expression in the mitochondria and cytosol. Taken together, these results suggested that picroside II has the ability to inhibit cytochrome $c$ release from the mitochondria to cytosol.
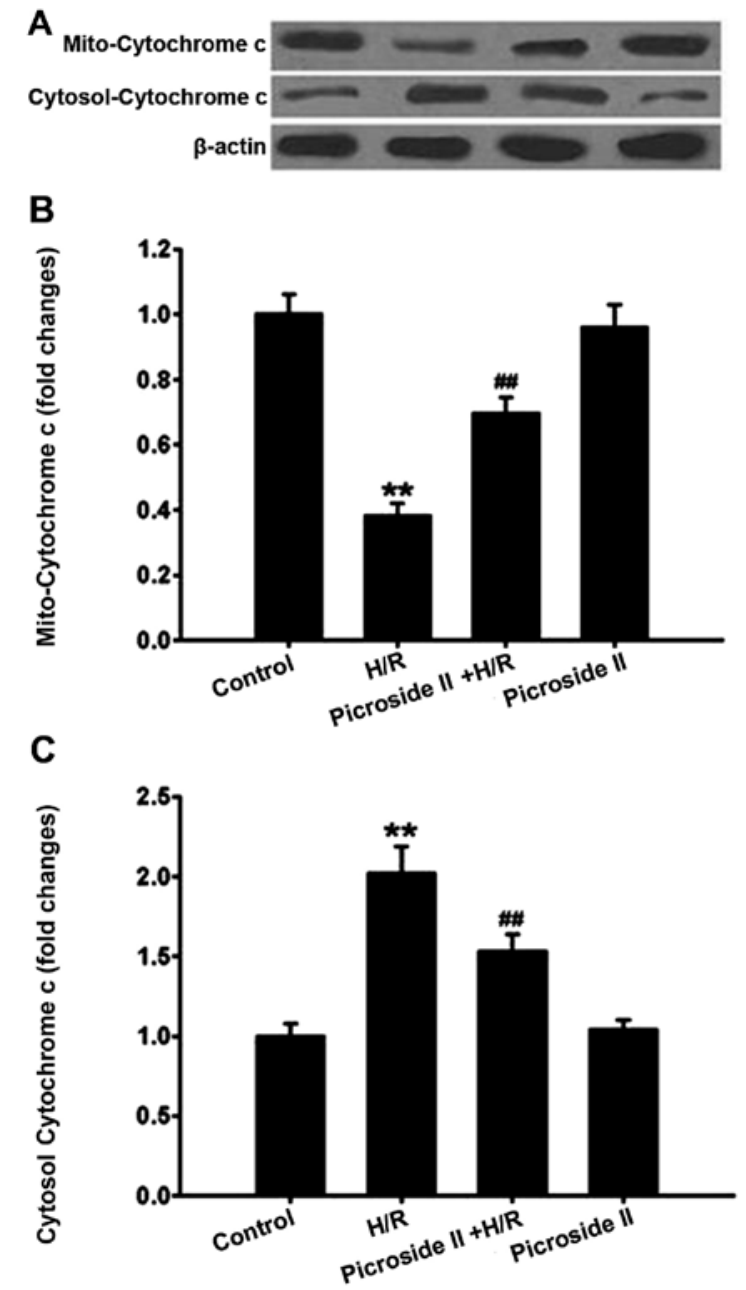

Figure 7. Effect of picroside II on H/R-induced protein expression of cytochrome $c$ in the mitochondria and cytosol in H9c2 cells. (A) Representative image of cytochrome $c$ protein expression in the mitochondria and cytosol by western blotting. Optical density of cytochrome $c$ protein bands in (B) the mitochondria and (C) cytosol. Data are expressed as mean \pm standard error of the mean and represents four independent experiments. ${ }^{* *} \mathrm{P}<0.01$ vs. control; ${ }^{\# \#} \mathrm{P}<0.01$ vs. H/R. H/R, hypoxia/reoxygenation.

\section{Discussion}

In the present study, the protective effect of picroside II on $\mathrm{H} / \mathrm{R}$-induced cardiomyocyte apoptosis and the underlying mechanisms in the $\mathrm{H} 9 \mathrm{c} 2$ rat cardiomyocyte cell line were investigated. The results indicated that pretreatment with picroside II was able to protect cardiomyocytes from H/R-induced apoptosis, which was associated with decreasing the opening degree of mPTP, increasing mitochondrial membrane potential, inhibiting cytochrome $c$ release from the mitochondria to the cytosol and downregulating caspase-3 expression and activity through a mechanism involving a decrease in ROS production.

Myocardial I/R injury is a common clinical problem and may result in serious consequences, including heart failure and mortality. Although the exact pathophysiological mechanism leading to myocardial $\mathrm{I} / \mathrm{R}$ injury is not fully understood, oxidative damage has long been considered a key factor in the initiation of I/R injury (21). Preclinical studies also consistently show that inhibition of ROS production can significantly alleviate I/R-induced injury $(22,23)$. Cardiomyocyte apoptosis 
is well-recognized to play a crucial role in $\mathrm{I} / \mathrm{R}$ injury $(5,6)$. Therefore, inhibition of cardiomyocyte apoptosis may be a potential strategy to prevent I/R-induced injury. Although the causes of cardiomyocyte apoptosis induced by I/R are complicated, it is widely accepted that excessive production of ROS during I/R play an important role in cardiomyocyte apoptosis $(7,8)$.

The mitochondrial compartment is the main target of intracellular ROS as they are particularly rich in polyunsaturated fatty acids $(24,25)$. Increased ROS can result in serious dysfunction of mitochondrion, which is the main cause of I/R-induced cardiomyocyte apoptosis (6). A pivotal factor mediating mitochondrial dysfunction is the long-lasting opening of MPTP, a large non-selective conductance pore located in the inner membrane of mitochondria $(15,26)$. Under physiological conditions, the transient mPTP opening may occur in normal cells (27). However, under pathological conditions the long-lasting mPTP opening allows protons into mitochondria, and subsequently results in the uncoupling of the electron respiratory chain and the decrease in mitochondrial membrane potential (26). The decrease in mitochondrial membrane potential is one of the earliest events in cell apoptosis (28). The long-lasting mPTP opening increases mitochondrial permeability and allows ions and solutes with molecular weights of $<1.5 \mathrm{kDa}$ in cytoplasm freely entering the mitochondria, leading to mitochondrial matrix swelling and loss of critical electrochemical gradients (29). Another adverse consequence of the mPTP opening is the cytochrome $c$ release from the mitochondria to the cytosol. Cytochrome $c$ is a well-known pro-apoptotic factor, and the translocation plays an important role in apoptosis by activating caspase cascade reactions. In the cytosol, cytochrome $c$ binds the apoptotic factor, Apaf-1, and induces Apaf-1 oligomerization, resulting in recruitment and activation of procaspase-9 (30). In turn, activated caspase-9 cleaves and activates caspase-3 (a classical executioner caspase), which terminally leads to cardiomyocyte apoptosis (31). Inhibition of the mPTP opening by cyclosporine A (an inhibitor of mPTP) or genetic deletion of the gene encoding CypD can protect against I/R-induced myocardial injury $(27,32)$. The above studies indicated that the sustained opening of the MPTP plays an important role in cell apoptosis. Strong evidence indicates that overproduction of ROS in the I/R period is the primary triggers of the mPTP opening (15). Decrease in ROS production is able to markedly inhibit the opening of mPTP (26). Therefore, inhibition of ROS overgeneration may be a promising strategy for prevention of I/R-induced cardiomyocyte apoptosis through suppressing the mPTP opening.

Picroside II, an iridoid glucoside extracted from Picrorhiza scrophulariiflora Pennell, has been reported to have multiple pharmacological actions, such as anti-inflammation (33) and neuroprotective effect (34). Most recently, it has been reported that picroside II has the protective effect on H/R-induced cardiomyocyte apoptosis (20), but the underlying mechanisms are not fully understood. As mentioned previously, picroside II scavenged oxygen free radical, protected normal constructions of mitochondria membrane $(19,35)$ and increased ROS-induced mitochondrial dysfunction during I/R, which plays an important role in cardiomyocyte apoptosis. Therefore, we hypothesized that the protective mechanism of picroside II on H/R-induced cardiomyocyte apoptosis may be associated with ameliorating mitochondrial function by a decrease in ROS production. In the present study, pretreatment of picroside II markedly inhibited H/R-induced cardiomyocyte apoptosis concomitantly with a decrease in ROS production. The further study showed that mitochondrial function was also significantly ameliorated, which was indicated by the decreased mPTP opening, increased mitochondrial membrane potential and decreased cytochrome $c$ release from mitochondria to cytosol.

In conclusion, the present study suggests that picroside II is able to inhibit H/R-induced cardiomyocyte apoptosis through ameliorating mitochondrial function involving a mechanism of decrease in ROS production.

\section{Acknowledgements}

The present study was supported by the Guangxi Nature Science Foundation of China (grant no. 2013GXNSFBA019126 to J. Z. Li), the Bureau of Public Health of Guangxi Province (grant no. Z2013206) and the National Nature Science Foundation of China (grant no. 81101476 to S. Y. Yu).

\section{References}

1. Dianat M,Esmaeilizadeh M, Badavi M, Samarbaf-Zadeh AR and Naghizadeh B: Protective effects of crocin on ischemia-reperfusion induced oxidative stress in comparison with vitamin $\mathrm{E}$ in isolated rat hearts. Jundishapur J Nat Pharm Prod 9: e17187, 2014.

2. Rodrigo R, Libuy M, Feliú F and Hasson D: Molecular basis of cardioprotective effect of antioxidant vitamins in myocardial infarction. Biomed Res Int 2013: 437613, 2013.

3. Wongcharoen W, Jai-Aue S, Phrommintikul A, Nawarawong W, Woragidpoonpol S, Tepsuwan T, Sukonthasarn A, Apaijai N and Chattipakorn N: Effects of curcuminoids on frequency of acute myocardial infarction after coronary artery bypass grafting. Am J Cardiol 110: 40-44, 2012.

4. Syrjälä SO, Tuuminen R, Nykänen AI, Raissadati A, Dashkevich A, Keränen MA, Arnaudova R, Krebs R, Leow CC, Saharinen P, Alitalo K and Lemström KB: Angiopoietin-2 inhibition prevents transplant ischemia-reperfusion injury and chronic rejection in rat cardiac allografts. Am J Transplant 14: 1096-1108, 2014.

5. Liu Y, Yang H, Song L, Li N, Han QY, Tian C, Gao E, Du J, Xia YL and Li HH: AGGF1 protects from myocardial ischemia/reperfusion injury by regulating myocardialapoptosis and angiogenesis. Apoptosis 19: 1254-1268, 2014.

6. Wang Y, Li X, Wang X, Lau W, Wang Y, Xing Y, Zhang X, Ma X and Gao F: Ginsenoside Rd attenuates myocardial ischemia/reperfusion injury via Akt/GSK-3 $\beta$ signaling and inhibition of the mitochondria-dependent apoptotic pathway. PLoS One 8: e70956, 2013.

7. Wu B, Meng K, Ji Q, Yu K, Zhao X, Tony H, Liu Y, Zhou Y, Chang C, Zhong Y, Zhu Z, Zhang W, Mao X and Zeng Q: Interleukin-37 ameliorates myocardial ischaemia/reperfusion injury in mice. Clin Exp Immunol 176: 438-451, 2014.

8. Peng YW, Buller CL and Charpie JR: Impact of N-acetylcysteine on neonatal cardiomyocyte ischemia-reperfusion injury. Pediatr Res 70: 61-66, 2011.

9. Li JZ, Yu SY, Wu JH, Shao QR and Dong XM: Paeoniflorin protects myocardial cell from doxorubicin-induced apoptosis through inhibition of NADPH oxidase. Can J Physiol Pharmacol 90: 1569-1575, 2012.

10. Teshima Y, Takahashi N, Nishio S, Saito S, Kondo H, Fukui A Aoki K, Yufu K, Nakagawa M and Saikawa T: Production of reactive oxygen species in the diabetic heart. Roles of mitochondria and NADPH oxidase. Circ J 78: 300-306, 2014.

11. Matsushima S, Tsutsui H and Sadoshima J: Physiological and pathological functions of NADPH oxidases during myocardial ischemia-reperfusion. Trends Cardiovasc Med 24: 202-205, 2014.

12. Dröse S, Brandt U and Wittig I: Mitochondrial respiratory chain complexes as sources and targets of thiol-based redox-regulation. Biochim Biophys Acta 1844: 1344-1354, 2014. 
13. Loor G, Kondapalli J, Iwase H, Chandel NS, Waypa GB Guzy RD, Vanden Hoek TL and Schumacker PT: Mitochondrial oxidant stress triggers cell death in simulated ischemia-reperfusion. Biochim Biophys Acta 1813: 1382-1394, 2011.

14. Ma WW, Hou CC, Zhou X, Yu HL, Xi YD, Ding J, Zhao X and Xiao R: Genistein alleviates the mitochondria-targeted DNA damage induced by $\beta$-amyloid peptides $25-35$ in C6 glioma cells. Neurochem Res 38: 1315-1323, 2013.

15. Li J, Umar S, Iorga A, Youn JY, Wang Y, Regitz-Zagrosek V, Cai $\mathrm{H}$ and Eghbali M: Cardiac vulnerability to ischemia/reperfusion injury drastically increases in late pregnancy. Basic Res Cardiol 107: 271, 2012.

16. Cui J, Li Z, Qian LB, Gao Q, Wang J, Xue M, Lou XE, Bruce IC, Xia Q and Wang HP: Reducing the oxidative stress mediates the cardioprotection of bicyclol against ischemia-reperfusion injury inrats. J Zhejiang Univ Sci B 14: 487-495, 2013.

17. Meng FJ, Hou ZW, Li Y and Yu B: The protective effect of picroside II against hypoxia/reoxygenation injury in neonatal rat cardiomyocytes. Pharm Biol 50: 1226-1232, 2012.

18. Li T, Liu JW, Zhang XD, Guo MC and Ji G: The neuroprotective effect of picroside II from hu-huang-lian against oxidative stress. Am J Chin Med 35: 681-691, 2007.

19. Gao H and Zhou YW: Anti-lipid peroxidation and protection of liver mitochondria against injuries by picroside II. World J Gastroenterol 11: 3671-3674, 2005.

20. Meng FJ, Jiao SM and Yu B: Picroside II protects cardiomyocytes from hypoxia/reoxygenation-induced apoptosis by activating the PI3K/Aktand CREB pathways. Int J Mol Med 30: 263-270, 2012.

21. de Vries DK, Kortekaas KA, Tsikas D, Wijermars LG, van Noorden CJ, Suchy MT, Cobbaert CM, Klautz RJ, Schaapherder AF and Lindeman JH: Oxidative damage in clinical ischemia/reperfusion injury: a reappraisal. Antioxid Redox Signal 19: 535-545, 2013.

22. Pisarenko OI, Lankin VZ, Konovalova GG, Serebryakova LI, Shulzhenko VS, Timoshin AA, Tskitishvili OV, Pelogeykina YA and Studneva IM: Apelin-12 and its structural analog enhance antioxidant defense in experimental myocardial ischemia and reperfusion. Mol Cell Biochem 391: 241-250, 2014.

23. Ma S, Zhang Z, Yi F, Wang Y, Zhang X, Li X, Yuan Y and Cao F: Protective effects of low-frequency magnetic fields on cardiomyocytes from ischemia reperfusion injury via ROS and NO/ONOO-. Oxid Med Cell Longev 2013: 529173, 2013.

24. Gao C, Chen X, Li J, Li Y, Tang Y, Liu L, Chen S, Yu H, Liu L and Yao P: Myocardial mitochondrial oxidative stress and dysfunction in intense exercise: regulatory effects of quercetin. Eur J Appl Physiol 114: 695-705, 2014.
25. Seleznev K, Zhao C, Zhang XH, Song K and Ma ZA: Calcium-independent phospholipase A2 localizes in and protects mitochondria during apoptotic induction by staurosporine. J Biol Chem 281: 22275-22288, 2006

26. Yue R, Hu H, Yiu KH, Luo T, Zhou Z, Xu L, Zhang S, Li K and Yu Z: Lycopene protects against hypoxia/reoxygenation-induced apoptosis by preventing mitochondrial dysfunction in primary neonatal mouse cardiomyocytes. PLoS One 7: e50778, 2012.

27. Schriewer JM, Peek CB, Bass J and Schumacker PT: ROS-mediated PARP activity undermines mitochondrial function after permeability transition pore opening during myocardial ischemia-reperfusion. J Am Heart Assoc 2: e000159, 2013.

28. Wadia JS, Chalmers-Redman RM, Ju WJ, Carlile GW, Phillips JL, Fraser AD and Tatton WG: Mitochondrial membrane potential and nuclear changes in apoptosis caused by serum and nerve growth factor withdrawal: time course and modification by (-)-deprenyl. J Neurosci 18: 932-947, 1998.

29. Perrelli MG, Pagliaro P and Penna C: Ischemia/reperfusion injury and cardioprotective mechanisms: Role of mitochondria and reactive oxygen species. World J Cardiol 3: 186-200, 2011.

30. Martin MC, Allan LA, Lickrish M, Sampson C, Morrice N and Clarke PR: Protein kinase A regulates caspase-9 activation by Apaf-1 downstream of cytochrome c. J Biol Chem 280 15449-15455, 2005.

31. Li P, Nijhawan D, Budihardjo I, Srinivasula SM, Ahmad M, Alnemri ES and Wang X: Cytochrome $\mathrm{c}$ and dATP-dependent formation of Apaf-1/caspase-9 complex initiates an apoptotic protease cascade. Cell 91: 479-489, 1997.

32. Gedik N, Heusch G and Skyschally A: Infarct size reduction by cyclosporine $\mathrm{A}$ at reperfusion involves inhibition of the mitochondrial permeability transition pore but does not improve mitochondrial respiration. Arch Med Sci 9: 968-975, 2013.

33. Guo Y, Xu X, Li Q, Li Z and Du F: Anti-inflammation effects of picroside 2 in cerebral ischemic injury rats. Behav Brain Funct 6 : 43, 2010.

34. Zhao L, Guo Y, Ji X and Zhang M: The neuroprotective effect of picroside II via regulating the expression of myelin basic protein after cerebral ischemia injury in rats. BMC Neurosci 15: 25 , 2014.

35. Cao Y, Liu JW, Yu YJ, Zheng PY, Zhang XD, Li T and Guo MC: Synergistic protective effect of picroside II and NGF on PC12 cells against oxidative stress induced by $\mathrm{H}_{2} \mathrm{O}_{2}$. Pharmacol Rep 59: 573-579, 2007. 